\title{
Impacts of Transceiver Configuration on Ultraviolet Communication
}

\author{
Tao Shan1, Jianshe Ma², Tianfeng Wu', Zanqiu Shen', Ping $\mathrm{Su}^{2}$ \\ ${ }^{1}$ Department of Precision Instrument, Tsinghua University, Beijing, China \\ ${ }^{2}$ Tsinghua Shenzhen International Graduate School, Tsinghua University, Shenzhen, China \\ Email:su.ping@sz.tsinghua.edu.cn
}

How to cite this paper: Shan, T., Ma, J.S., Wu, T.F., Shen, Z.Q. and Su, P. (2020) Impacts of Transceiver Configuration on Ultraviolet Communication. Optics and Photonics Journal, 10, 89-94. https://doi.org/10.4236/opj.2020.106008

Received: January 19, 2020

Accepted: June 26, 2020

Published: June 29, 2020

\begin{abstract}
In the channel estimation for ultraviolet communication, the single scattering power is usually used to approximate the received total power. This approximation error is affected by the transceiver configuration. Here, we employ the proportion of received single scattering power in received total power to indicate the approximation error of the single scattering model in different configurations. This is useful for reducing the approximation error by selecting a more appropriate transceiver configuration.
\end{abstract}

\section{Keywords}

Ultraviolet Communication, Single Scattering, Non-Line-of-Sight

\section{Introduction}

With the recent advances of ultraviolet (UV) source and detectors in the solar blind wavelength regime, UV communication system has attracted increasing attention. The non-line-of-sight (NLOS) channel modeling of UV communication has gradually become the core issue [1]. One valid way for channel modeling is to establish the single scattering channel model by assuming that ultraviolet photons traveling in the medium between the source and the detector are scattered only once in short-range cases [2] [3] [4]. Another way is to establish the multiple scattering channel model based on the Monte-Carlo method [5] [6]. As the communication range increases, single scattering model was modified by applying the atmospheric turbulence theory for the increasing atmospheric effect on the communication performance [7]. Unfortunately, the increase of communication range also decreases the proportion of single scattering power in received total power. This proportion reflects the approximation error of single 
scattering model itself. Since the proportion is affected by system geometry, the impacts of transceiver configuration on this proportion need to be analyzed in UV communication.

In this work, the proportion of the received single scattering power in the received total power is employed as an indicator to evaluate the approximation error and the effectiveness of the single scattering model under different transceiver configurations. The simulation results demonstrate that this proportion decreases with the increase of elevation angle, field-of-view (FOV) angle and the communication range. Thus, the effective range of single scattering model is limited by the transceiver configuration. We also find that the approximation error of the single scattering model in UV communication can be reduced by selecting a more appropriate configuration.

\section{Methodology}

Figure 1 shows a typical NLOS UV communication geometry. The configuration parameters are defined as follows: $r$ is the baseline separation between transmitter $(\mathrm{T})$ and receiver $(\mathrm{R}) .\left(\theta_{T}, \beta_{T}\right)$ and $\left(\theta_{R}, \beta_{R}\right)$ are the elevation angle and the divergence angle of beam and FOV, respectively. $\theta_{S}=\theta_{T}+\theta_{R}$ is the scattering angle between the photon forward direction and the observation direction. In the NLOS communication as shown in Figure 1, single scattering model is widely used to estimate channel performance. However, the power received at $\mathrm{R}$ is a combination of the scattering power of all scattering orders, and the received scattering power for each order changes as the transceiver configuration changes. Thus, the proportion of received single scattering power $P_{1}$ in received total power $P_{\text {all }}$ can be an indicator to describe the approximation error and show the validity of single scattering model in different configurations. Higher proportion indicates the single scattering model in certain transceiver configuration is more accurate and more effective. The relationship between proportion and approximation error is error $=10 \log _{10}\left(P_{\text {all }} / P_{1}\right) .80 \%$ in proportion indicates that the error of approximating multiple scattering power with single scattering power is $1 \mathrm{~dB}$.

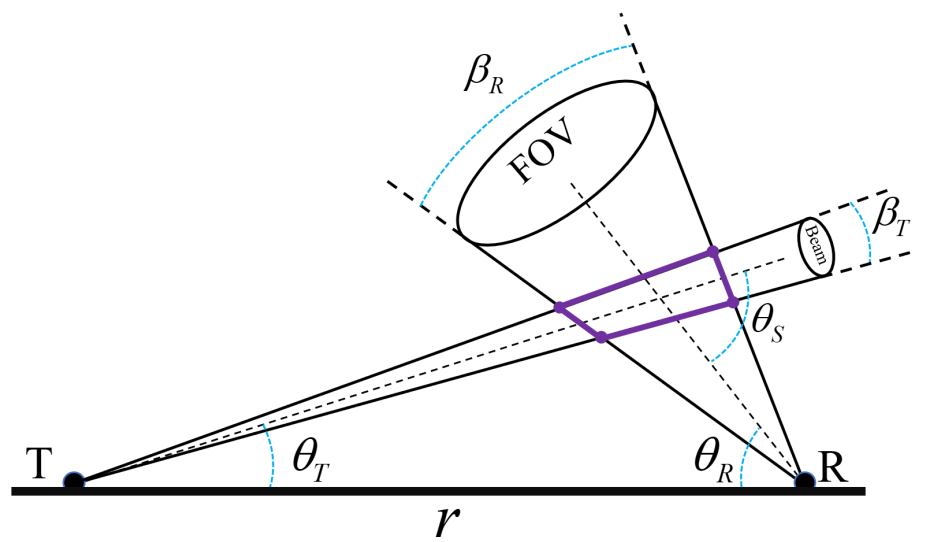

Figure 1. NLOS UV communication geometry. 


\section{Simulation Analysis}

We take multiple scattering model [6] to simulate the proportion and assume the received total power is equal to the summation of the scattering powers of the first four scattering orders. The model parameters are selected as: $\left(k_{e}, k_{s}\right)=(0.802,0.550) \mathrm{km}^{-1}, A_{r}=1.77 \mathrm{~cm}^{2}, \lambda=260 \mathrm{~nm}, \gamma=0.017$, $g=0.72, f=0.5, \quad P_{e}=30 \mathrm{~mW}$ [3].

First, the proportion under different communication ranges $r$ and transmitter elevation angles $\theta_{T}$ are simulated by multiple scattering model [6], as shown in Figure $2\left(\beta_{T}=1^{\circ}, \theta_{R}=65^{\circ}, \beta_{R}=30^{\circ}\right)$. We can find that the proportion decreases when $r$ or $\theta_{T}$ increases. That is because the longer communication range and larger elevation angle both result in longer transmitting paths, thus the higher probability of photon extinction. In addition, the larger transmitter elevation angle also causes the larger scattering angle $\theta_{S}$, which means fewer photons can arrive at the receiver by single scattering. In Figure 2 , as the $\theta_{T}$ is equal to $5^{\circ}$, the decline in the proportion from $100 \mathrm{~m}$ to $1500 \mathrm{~m}$ is $11 \%$. And this decline in the proportion is rising as $\theta_{T}$ increases: the decline in the proportion from 100 $\mathrm{m}$ to $1500 \mathrm{~m}$ is $49 \%$ when $\theta_{T}$ is $40^{\circ}$. As shown in Figure 2 , even the communication range is $1500 \mathrm{~m}$, the proportion still reach $80 \%$ as long as $\theta_{T}$ is less than $10^{\circ}$. However, when the $\theta_{T}$ is $35^{\circ}$, the proportion drops to $77 \%$ (i.e., the proportion is smaller than $80 \%$ ) as the communication range reaches $500 \mathrm{~m}$. In $1500 \mathrm{~m}$, the proportion drops to $49 \%$ corresponding to $3.1 \mathrm{~dB}$ in approximation error. Therefore, in system design, we can select a lower transmitter elevation angle to increase the proportion and to reduce approximation error in single scattering model. When maximum tolerable error is determined, we can enlarge effective range of single scattering model by reducing the elevation angles.

Second, Figure 3 shows the relationships between the proportion with the receiver elevation angle and the communication range $\left(\beta_{T}=1^{\circ}, \theta_{T}=25^{\circ}, \beta_{R}=30^{\circ}\right)$. Similar to the impacts that transmitter elevation angle exerts on the proportion, the rise of receive elevation angle also causes the increase of it.

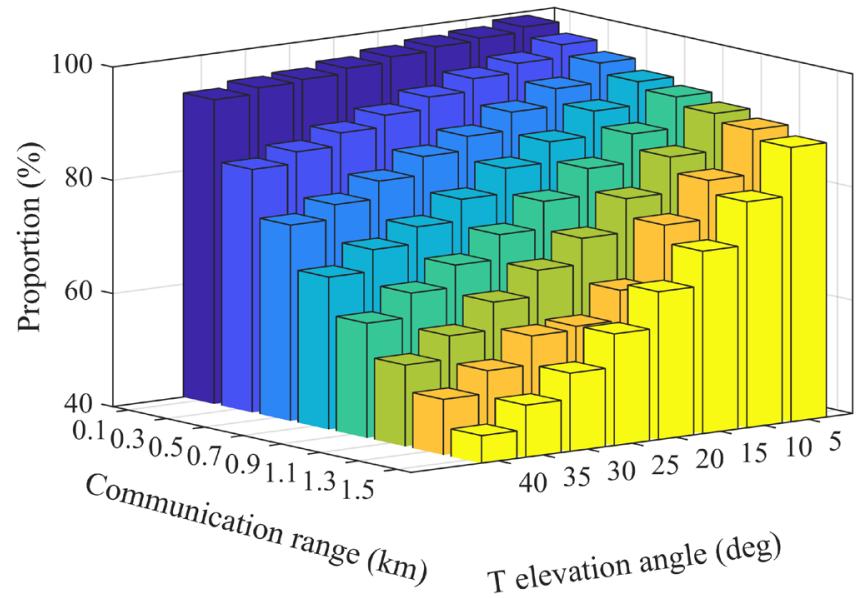

Figure 2. Proportion versus communication range and transmitter elevation angle. 


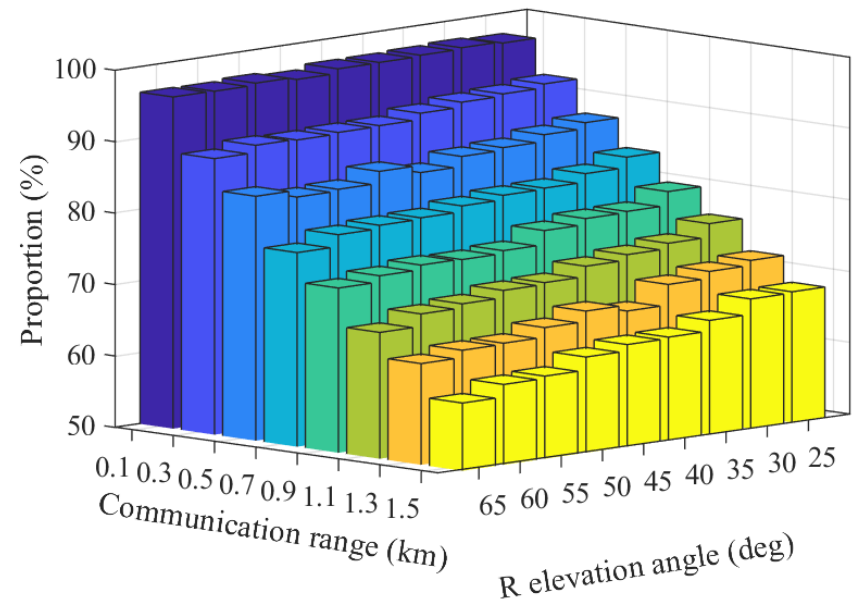

Figure 3. Proportion versus communication range and receiver elevation angle.

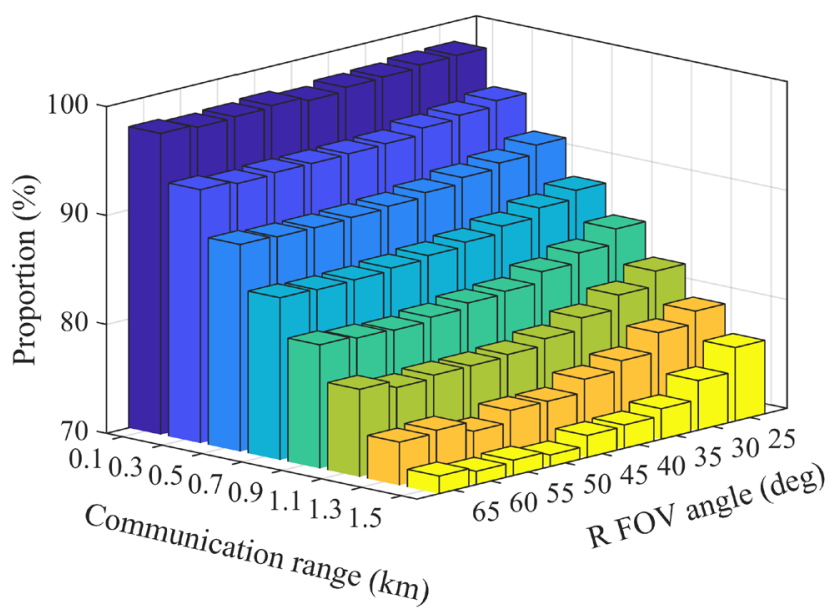

Figure 4. Proportion versus communication range and receiver FOV angle.

Third, in addition to two elevation angles and communication range, the changes in receiver FOV (i.e., $\beta_{R}$ ) also affect the proportion, as shown in Figure $4\left(\beta_{T}=1^{\circ}, \theta_{T}=15^{\circ}, \theta_{R}=40^{\circ}\right)$. With $\beta_{R}$ increasing from $25^{\circ}$ to $70^{\circ}$, the proportion first decreases to the minimum and then increases slightly. The maximal proportion appears when $\beta_{R}$ is at the a minimum (i.e., $\beta_{R}$ is equal to $\left.25^{\circ}\right)$. When we select a larger FOV angle to receive more optical power [8], the proportion decreases and single scattering model causes more absolute error. Thus, smaller FOV angle is needed to improve the effectiveness of single scattering model.

Based on the preceding analysis, the proportion of received single scattering power in received total power increases with the decrease of elevation angles and FOV angle. Three kinds of NLOS transceiver configuration in small elevation angle and small FOV case are shown in Figure 5: (i) Both elevation angles are small $\left(\beta_{T}=1^{\circ}, \beta_{R}=25^{\circ}, \theta_{T}=15^{\circ}, \theta_{R}=15^{\circ}\right)$; (ii) Only transmitter elevation is small $\left(\beta_{T}=1^{\circ}, \beta_{R}=25^{\circ}, \theta_{T}=15^{\circ}, \theta_{R}=65^{\circ}\right)$; (iii) Only receiver elevation is small 
(i)

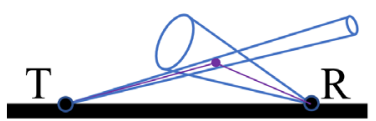

(ii)

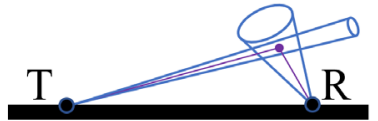

(iii)

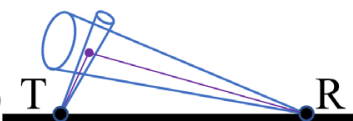

Figure 5. Three NLOS transceiver configurations in small elevation angle: (i) Both $\theta_{T}$ and $\theta_{R}$ are small; (ii) Only $\theta_{T}$ is small; (iii) Only $\theta_{R}$ is small.

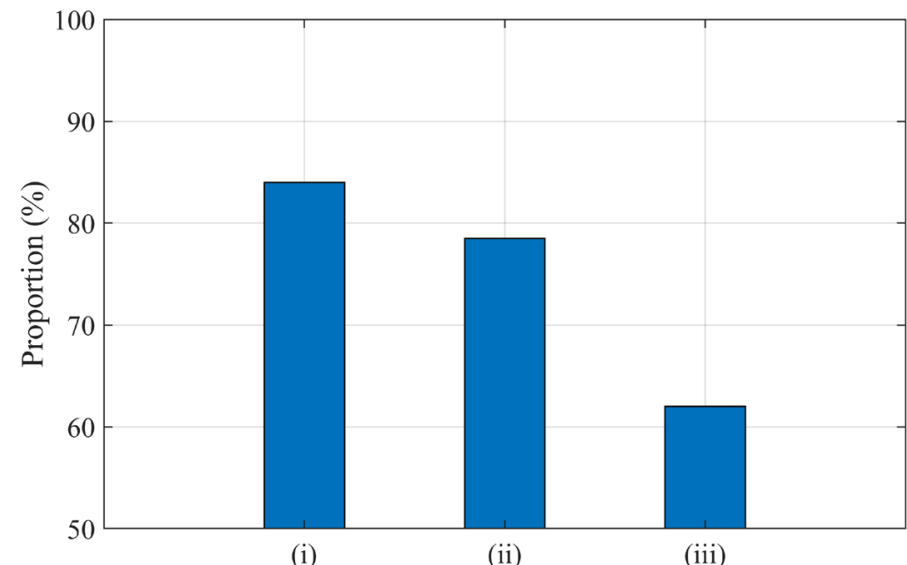

Figure 6. Proportion and path loss in three configurations of small elevation angle.

$\left(\beta_{T}=1^{\circ}, \beta_{R}=25^{\circ}, \theta_{T}=65^{\circ}, \theta_{R}=15^{\circ}\right)$. Figure 6 depicts the proportion in these configurations when the communication range is $1250 \mathrm{~m}$. The configuration (i) has the highest proportion because of the shortest effective path. The proportion in this configuration is $84 \%$ corresponding to $0.7 \mathrm{~dB}$ in approximation error, which is negligible when communication range is $1250 \mathrm{~m}$. In addition, the performance in the configuration (ii) is much better than that in the configuration (iii), even though the length of central paths in both configurations is equal. Thus, in the system design, the priority order of these three NLOS transceiver configuration in small elevation angle is (i) $>$ (ii) $>$ (iii).

\section{Conclusions}

The proportion of the received single scattering power in the received total power is employed to indicate the impacts of transceiver configuration. We use the proportion to analyze the approximation error and the effective range of single scattering model. The results show that the effective range of single scattering model is limited by the transceiver configuration. Furthermore, the approximation error of single scattering model can be negligible in the case of small elevation angles and small FOV, even in long range communication system. 


\section{Funding}

The Basic Research Program of Shenzhen (JCYJ20170412171744267).

\section{Conflicts of Interest}

The authors declare no conflicts of interest regarding the publication of this paper.

\section{References}

[1] Yuan, R.Z. and Ma, J.S. (2016) Review of Ultraviolet Non-Line-of-Sight Communication. China Communications, 13, 63-75. https://doi.org/10.1109/CC.2016.7513203

[2] Luettgen, M., Shapiro, J. and Reilly, D. (1991) Non-Line-of-Sight Single-Scatter Propagation Model. JOSA A, 8, 1964-1972. https://doi.org/10.1364/JOSAA.8.001964

[3] Wu, T.F., Ma, J.S., Su, P., Yuan, R.Z. and Cheng, J. (2019) Modeling of Short-Range Ultraviolet Communication Channel Based on Spherical Coordinate. IEEE Communication Letters, 23, 242-245. https://doi.org/10.1109/LCOMM.2018.2890518

[4] Wu, T.F., Ma, J.S., Yuan, R.Z., Su, P. and Cheng, J. (2019) Single-Scatter Model for Short-Range Ultraviolet Communication in a Narrow Beam Case. IEEE Photonics Technology Letters, 31, 265-268. https://doi.org/10.1109/LPT.2019.2891129

[5] Ding, H.P., Chen, G. Majumdar, A.K., Sadler, B.M. and Xu, Z.Y. (2009) Modeling of Non-Line-of-Sight Ultraviolet Scattering Channels for Communication. IEEE Journal on Selected Areas in Communications, 27, 1534-1544. https://doi.org/10.1109/JSAC.2009.091203

[6] Drost, R., Moore, T. and Sadler, B.M. (2011) UV Communications Channel Modeling Incorporating Multiple Scattering Interactions. JOSA A, 28, 686-695. https://doi.org/10.1364/JOSAA.28.000686

[7] Ding, H.P., Chen, G., Majumdar, A.K., Sadler, B.M. and Xu, Z.Y. (2011) Turbulence Modeling for Non-Line-of-Sight Ultraviolet Scattering Channels. Proceedings of SPIE-The international Society for Optical Engineering, 8038, 73-91. https://doi.org/10.1117/12.889049

[8] Shen, Z.Q., Ma, J.S., Shan, T. and Wu, T.F. (2019) Modeling of Ultraviolet Scattering Propagation and Its Applicability Analysis. Optics Letters, 44, 4953-4956. https://doi.org/10.1364/OL.44.004953 